\title{
Photorefraktive Keratektomie bei Keratokonus
}

\section{Deepali Sandeep Tambe Anders Ivarsen Jesper Hjortdal}

Department of Ophthalmology, Aarhus University Hospital, Aarhus, Dänemark

\section{Schlüsselwörter}

Photorefraktive Keratektomie · Keratokonus · Refraktionschirurgie

\section{Zusammenfassung}

Zielsetzung: Beurteilung der Wirksamkeit und Sicherheit der topografiegeführten photorefraktiven Keratektomie (PRK) bei Keratokonus und Einschätzung des Risikos einer nachfolgenden Progression.

Methoden: Hierbei handelt es sich um eine retrospektive Nachbeobachtungsstudie. Zwischen 1998 und 2013 wurden 28 Augen von 23 Patienten (Alter 17-60 Jahre) mit Keratokonus im Stadium 1-3 mit einer topografiegeführten PRK behandelt. Vor dem Eingriff, 3 Monate danach und bei einer Langzeit-Nachuntersuchung im Median 7 Jahre nach dem Eingriff wurden jeweils eine Messung des korrigierten Fernvisus (corrected distance visual acuity; CDVA), Keratometrie, Pachymetrie und Hornhaut-Topografie durchgeführt. Postoperative Komplikationen einschließlich späterer Keratoplastik wurden dokumentiert.

Ergebnisse: Bis zur Langzeit-Nachuntersuchung im Median 7 Jahre (Bereich 3-10 Jahre) nach der PRK war bei 5 der 28 Augen (18\%) eine Hornhaut-Transplantation vorgenommen worden. Vier Augen standen für die Nachuntersuchung nicht zur Verfügung. Bei den verbleibenden 19 Augen war der CDVA in 16 Augen (84,3\%) verbessert, in 2 Augen (10,5\%) verschlechtert und in 1 Auge (5,2\%) unverändert. Damit hatte sich der durchschnittliche CDVA von 0,49 logMAR vor der PRK auf 0,27 logMAR nach 3 Monaten und 0,24 logMAR bei der Langzeit-Nachuntersuchung verbessert. Das mittlere sphärische Äquivalent ging von $-6,2$ auf $-3,7$ dpt nach 3 Monaten und -2,1 dpt bei der Langzeit-Nachuntersuchung zurück. Ebenso verringerte sich der mittlere Zylinderwert von -4,2 auf -3,0 dpt nach 3 Monaten und bei der Langzeit-Nachuntersuchung. Schlussfolgerung: Die topografiegeführte PRK stellt bei Keratokonus potenziell eine wirksame Option zur Reduktion von Myopie und Astigmatismus sowie eine vorläufige oder dauerhafte Alternative zur Keratoplastik bei Patienten mit Keratokonus und Kontaktlinsen-Unverträglichkeit dar. In der hier vorgestellten Studie wurde ein geringes Risiko für eine Progression des Keratokonus nach einer PRK festgestellt.

(c) 2016 S. Karger GmbH, Freiburg

\section{Einleitung}

Der Keratokonus ist gekennzeichnet durch eine fortschreitende Vorwölbung und Ausdünnung der Hornhaut, die zu irregulärem Astigmatismus und einer Beeinträchtigung des Sehvermögens führt $[1,2]$. In den letzten 2 Jahrzehnten sind verschiedene Neuerungen für die visuelle Rehabilitation bei Keratokonus entwickelt worden, darunter neue Kontaktlinsen-Designs, die photorefraktive Keratektomie (PRK), die Kollagenvernetzung (Crosslinking), intrastromale corneale Ringsegmente (ICRS), phake
Intraokularlinsen sowie die penetrierende oder lamelläre Keratoplastik in fortgeschrittenen Stadien [2].

Derzeit werden bei der PRK topografiegeführte Ablationsprofile eingesetzt, um die Unregelmäßigkeiten der Hornhaut-Oberfläche zu mindern und den Visus zu verbessern. Mit einer Ausdünnung der Hornhaut einhergehende Erkrankungen schwächen die mechanische Belastbarkeit der betroffenen Hornhaut, was dafür spricht, dass photorefraktive Eingriffe bei Keratokonus kontrain-

\section{KARGER \\ Fax +497614520714 \\ information@karger.com}

www.karger.com (c) 2016 S. Karger GmbH, Freiburg

2297-0118/16/0021-0036\$39.50/0

Accessible online at:

www.karger.com/kop
Deepali Sandeep Tambe, MD

Department of Ophthalmology

Aarhus University Hospital

Noerrebrogade 44, 8000 Aarhus C, Dänemark

deepali.tambe@yahoo.com 
diziert sein könnten. Bisher liegen wenige Fallberichte vor, die diese Hypothese bestätigen [3]. Zwar gibt es Berichte über Hornhaut-Ektasien nach PRK bei Verdachtsfällen von Keratokonus [4], doch andere Studien haben ermutigende Ergebnisse mit alleiniger PRK erbracht [5-7].

Zwischen 1998 und 2013 führten wir bei 28 Augen von 23 Keratokonuspatienten eine PRK durch. Das Hauptziel war dabei, die Sehschärfe mit Brille bzw. Kontaktlinsen zu verbessern und so größere chirurgische Eingriffe wie z.B. eine Keratoplastik hinauszuzögern oder zu vermeiden.

Das Ziel der vorliegenden Studie war es, die langfristige Wirksamkeit und Sicherheit der PRK bei Keratokonus sowie das Risiko einer erneuten Krankheitsprogression nach der Excimer-LaserBehandlung zu beurteilen. Da bisher nur wenige Berichte zu dieser Frage vorliegen, sind wir der Ansicht, dass unsere Studie trotz des retrospektiven Designs und der relativ kleinen Fallzahl von Interesse ist.

\section{Patienten und Methoden}

\section{Teilnehmer}

Insgesamt 28 Augen von 23 Patienten (16 männlich und 7 weiblich; Durchschnittsalter 36 Jahre; Bereich 17-60 Jahre), bei denen zwischen 1998 und 2013 eine topografiegeführte PRK durchgeführt worden war, wurden retrospektiv aus elektronischen Patientenakten in der Abteilung für Ophthalmologie des Universitätsklinikums Aarhus, Dänemark, identifiziert. Alle Patienten waren zuvor von einem Augenarzt wegen schlechter Sehschärfe mit Brille und Unverträglichkeit gegenüber Kontaktlinsen zur Hornhaut-Transplantation überwiesen worden. Die Keratokonusdiagnose wurde anhand einer Kombination aus SpaltlampenBiomikroskopie, Hornhaut-Topografie, automatischer Keratometrie und Hornhaut-Pachymetrie gestellt. Präoperative und kurzfristig-postoperative Daten wurden aus den Patientenakten erhoben. Bei allen Patienten lag präoperativ ein Keratokonus im Stadium 1-3 laut Amsler-Krumeich-Klassifikation vor. Die Hornhaut-Trübung wurde mittels Fantes-Score beurteilt. Bei 5 Patienten waren beide Augen behandelt worden. Bei 2 Patienten wurde 1 erneute PRK durchgeführt, bei 1 Patienten erfolgten 2 PRK-Wiederholungsbehandlungen. Die Patienten wurden zu einer Langzeit-Nachuntersuchung in 2013/2014 eingeladen.

\section{Ein- und Ausschlusskriterien}

Die Gesamtzahl der behandelten Patienten betrug 23 (28 Augen). Die Auswertung der elektronischen Patientenakten ergab bei 5 Patienten (5 Augen), dass sie sich nach der PRK im weiteren Verlauf einer Keratoplastik unterzogen hatten. Diese 5 Fälle wurden als Versagen der PRK eingestuft, und die Patienten wurden nicht zu der Langzeit-Nachuntersuchung eingeladen. Zwei Patienten (2 Augen) waren unbekannt verzogen, und 1 Patient (2 Augen) war nicht an der Teilnahme an der Langzeit-Nachuntersuchung interessiert. Zwei Patienten (2 Augen) waren nicht zur Nachuntersuchung nach 3 Monaten erschienen. Somit wurden in die statistischen Analysen 26 Augen von 21 Patienten bei der Nachuntersu-
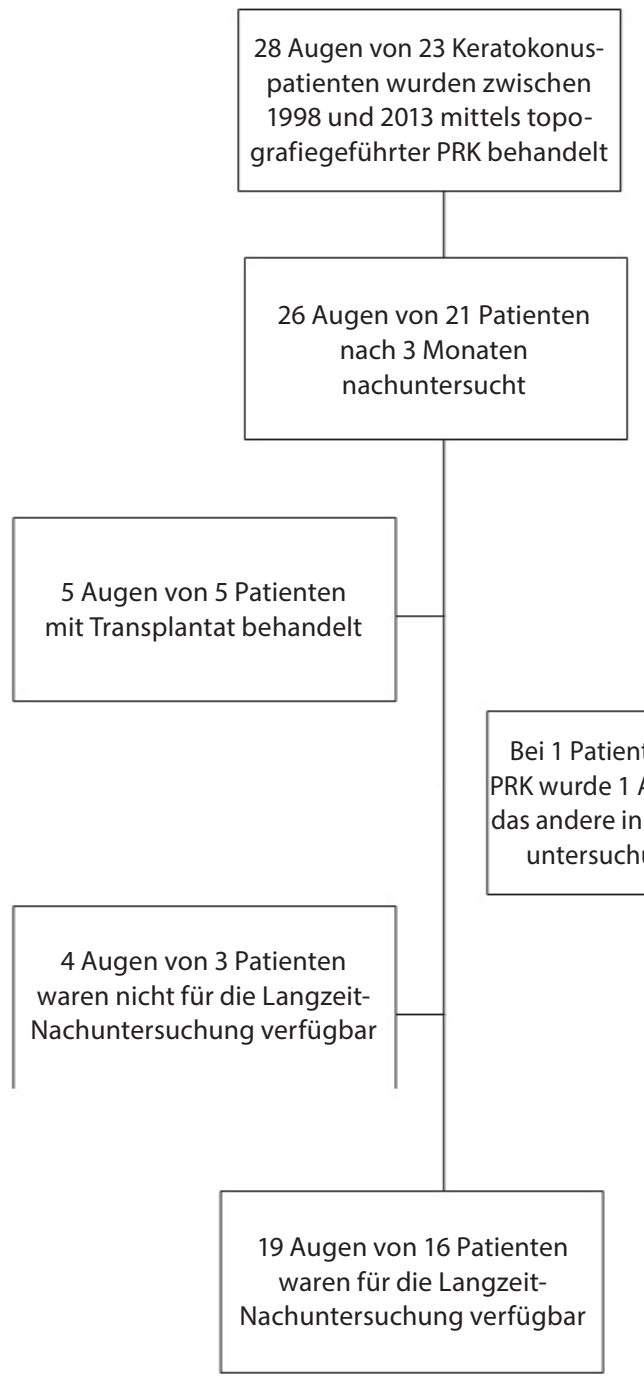

Abb. 1. Flussdiagramm der Gesamtheit der mit PRK behandelten Keratokonuspatienten sowie der Anzahl Patienten bei der Nachuntersuchung nach 3 Monaten und der Langzeit-Nachuntersuchung.

chung nach 3 Monaten und 19 Augen von 16 Patienten bei der Langzeit-Nachuntersuchung einbezogen (Abb. 1). Der Patient (2 Augen), der nicht an der Langzeit-Nachuntersuchung interessiert war, gab (im Telefongespräch) an, mit der Behandlung zufrieden zu sein. Daher beziehen sich die Daten zur Patientenzufriedenheit auf 21 Augen von 17 Patienten.

\section{Operationstechnik}

Anhand von Orbscan-II-Spaltbildern (Bausch and Lomb, Rochester, NY, USA) erstellte Topografiekarten wurden für die topografiegestützte Planung (TOSCA) der Ablation bei den zwischen 1998 und 2005 behandelten Patienten (12 Augen) verwendet. Bei den zwischen 2005 und 2013 behandelten Patienten (16 Augen) erfolgten die Vermessung mit dem ATLAS-Topografiesystem (Carl Zeiss Meditec, Jena, Deutschland) und die Behandlungsplanung mit dem CRS-Master-Softwaremodul. Die PRK wurde unter Oberflächenanästhesie durchgeführt. Alle Patienten wurden von 1 von 2 Chirurgen behandelt (Dr. Jesper Hjortdal 
oder Dr. Niels Ehlers, Universitätsklinikum Aarhus). Mit einem kreisförmigen Hornhaut-Marker wurde eine über der Pupille zentrierte 8-mm-Zone definiert. Mit einem chirurgischen Schwamm wurde kurz Alkohol appliziert und unmittelbar danach mit einem mit physiologischer Kochsalzlösung befeuchteten Schwamm abgewischt. Anschließend wurde das zentrale Epithel mit einem stumpfen Beaver-Messer entfernt. Die Photoablation mit dem Excimer-Laser wurde mit einem «Flying-Spot»-Excimer-Laser durchgeführt, entweder dem MEL-70 (12 Augen, 1998-2005) oder dem MEL-80 (16 Augen, 2005-2013) (Carl Zeiss Meditec). Der Durchmesser der optischen Zone betrug zwischen 6,0 und 6,5 mm. Die mittlere Ablationstiefe lag bei $82 \mu \mathrm{m}$ (Standardabweichung (SA) 17,8 $\mu \mathrm{m}$; Bereich 45-112 $\mu \mathrm{m}$ ). Bei 3 wiederholt behandelten Patienten (5 Augen) wurde $20 \mathrm{~s}$ lang topisches Mitomycin C 0,02\% angewandt, um postoperative Trübungen zu minimieren. Nach der Excimer-Laser-Behandlung wurden 1 Tropfen Cyclopentolat 1\% und 1 Tropfen Diclofenac 0,1\% sowie Chloramphenicol-Salbe appliziert.

\section{Postoperative Behandlung und Nachbeobachtung}

Die postoperative Behandlung umfasste Chloramphenicol-Augentropfen 0,5\% und Prednisolon-Augentropfen 0,5\% 4-mal täglich für 2 Wochen und danach 2-mal täglich für weitere 2 Wochen. Die Patienten wurden vor der Operation untersucht und zu Nachuntersuchungen 1, 3, 6, 9 und 12 Monate nach der Operation eingeladen. Bei allen Terminen erfolgte eine klinische Routineuntersuchung mit Spaltlampen-Biomikroskopie, Bestimmung des korrigierten Fernvisus (corrected distance visual acuity; CDVA), Pachymetrie und Hornhaut-Topografie mit einem TMS-1, TMS-3 (beide Tomey, Nagoya, Japan), ATLAS (Carl Zeiss Meditec) oder einer Pentacam HR (Oculus, Wetzlar, Deutschland). Bei der Langzeit-Nachuntersuchung im Median 7 Jahre nach dem Eingriff (Bereich 2-14 Jahre) wurden die Patienten nach ihrer Zufriedenheit mit der PRK-Behandlung in jedem Auge gefragt; hierbei gab es lediglich die Antwortmöglichkeiten «zufrieden» oder «unzufrieden». Die Sehschärfedaten wurden von Snellen-Äquivalent in $\log$ MAR umgerechnet. Als Hauptparameter zur Beurteilung des Verlaufs galten die Veränderung des CDVA-Werts, die Refraktion als sphärisches Äquivalent (SÄ), die zylindrische Refraktion, Veränderungen in der Keratometrie (Kmax; aus der steilsten Keratometermessung) sowie jegliche Formen von Therapieversagen (Notwendigkeit einer Keratoplastik oder zusätzlichen elektiven Operation). Die zentrale Hornhaut-Dicke (central corneal thickness; CCT) sowie das Auftreten von Hornhaut-Trübung wurden als Sekundärparameter betrachtet. Eine Progression des Keratokonus war definiert als Anstieg der steilsten Keratometriemessung bei gleichzeitiger Veränderung der subjektiven Refraktion, Topografie und Hornhaut-Dicke zwischen der Nachuntersuchung 3 Monate nach der PRK und der Langzeit-Nachuntersuchung.

\section{Statistische Auswertung}

Für die statistischen Analysen wurde die Software GraphPad Prism (Version 6.0d) verwendet. Die präoperativen, frühen post- operativen (Nachuntersuchung nach 3 Monaten) und späten postoperativen Parameterdaten wurden mittels gepaarter t-Tests verglichen. Hierbei galten $\mathrm{p}$-Werte $<0,05$ als statistisch signifikant.

\section{Ergebnisse}

\section{Progression des Keratokonus}

Bei der Langzeit-Nachuntersuchung zeigten 15 von 19 Augen (79\%) keine Keratokonusprogression. In 5 der insgesamt $28 \mathrm{Au}-$ gen (18\%) war jedoch in den Jahren nach der PRK eine Hornhaut-Transplantation vorgenommen worden, und bei 1 Patienten (3\%) war die Implantation von ICRS geplant. Der Altersmedian der transplantierten Patienten betrug zum Zeitpunkt der PRK 47 Jahre (Bereich 30-51 Jahre). Bei 3 der späteren Transplantatempfänger hatte die topografiegeführte PRK zunächst Wirkung gezeigt, später war jedoch eine Progression mit Entwicklung eines ausgeprägten Keratokonus eingetreten; dies geschah im Median 7 Jahre (Bereich 3-10 Jahre) nach dem Eingriff. Ein 4. Patient erhielt ein Transplantat, weil sich nach der PRK eine zentrale Trübung vom Schweregrad 2 entwickelt hatte, der Keratokonus war jedoch stabil. Beim 5. Patienten erfolgte die Transplantation, weil nach der PRK nicht die erwarteten Ergebnisse erreicht wurden.

\section{Subjektive Zufriedenheit}

Die Langzeit-Nachuntersuchung im Median 7 Jahre nach der PRK ergab, dass 13 Patienten (16 Augen) mit der PRK zufrieden und 4 Patienten (5 Augen) unzufrieden waren. Eine langfristige Zufriedenheit mit der PRK-Behandlung bestand also in Bezug auf mindestens 16 der 28 behandelten Augen (57\%).

\section{Unkorrigierter Fernvisus}

Der mittlere unkorrigierte Fernvisus verbesserte sich signifikant von 20/250 (1,1 logMAR) vor der Operation auf 20/100 (0,7 logMAR) bei der Langzeit-Nachuntersuchung ( $<0,05)$.

\section{Korrigierter Fernvisus}

In den 26 Augen, die nach 3 Monaten nachuntersucht wurden, hatte sich der CDVA in 21 Augen (81\%) um 1-7 Snellen-Zeilen verbessert, in den anderen 5 Augen (19\%) war er unverändert geblieben. In der Langzeit-Nachuntersuchung von 19 Augen hatte sich der CDVA in 16 Augen (84\%) um 1-4 Snellen-Zeilen verbessert, in 2 Augen (11\%) um 1-2 Snellen-Zeilen verschlechtert und war in 1 Auge (5\%) unverändert geblieben (Tab. 1). Der durchschnittliche CDVA verbesserte sich nach der topografiegeführten PRK signifikant von 20/60 (0,49 logMAR) vor der Operation auf 20/40 (0,27 logMAR) bei der 3-Monats- und der Langzeit-Nachuntersuchung.

\section{Refraktionswerte}

Die mittlere SÄ-Refraktion wurde signifikant reduziert: um 2,5 dpt nach 3 Monaten ( $\mathrm{p}<0,05)$ und um 4,1 dpt bei der LangzeitNachuntersuchung ( $p<0,05$; Tab. 2, Abb. 2b). In einem gepaarten 


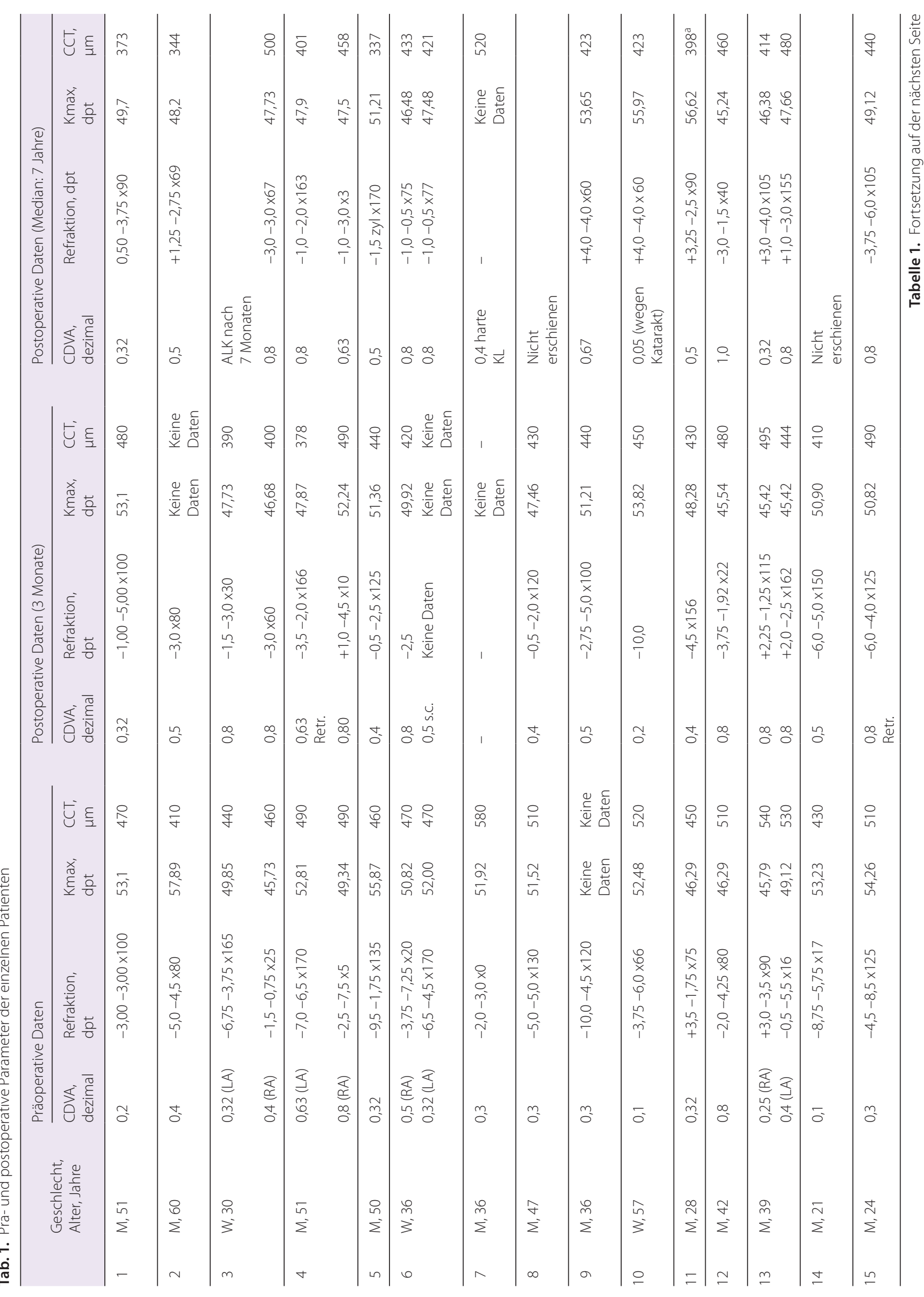




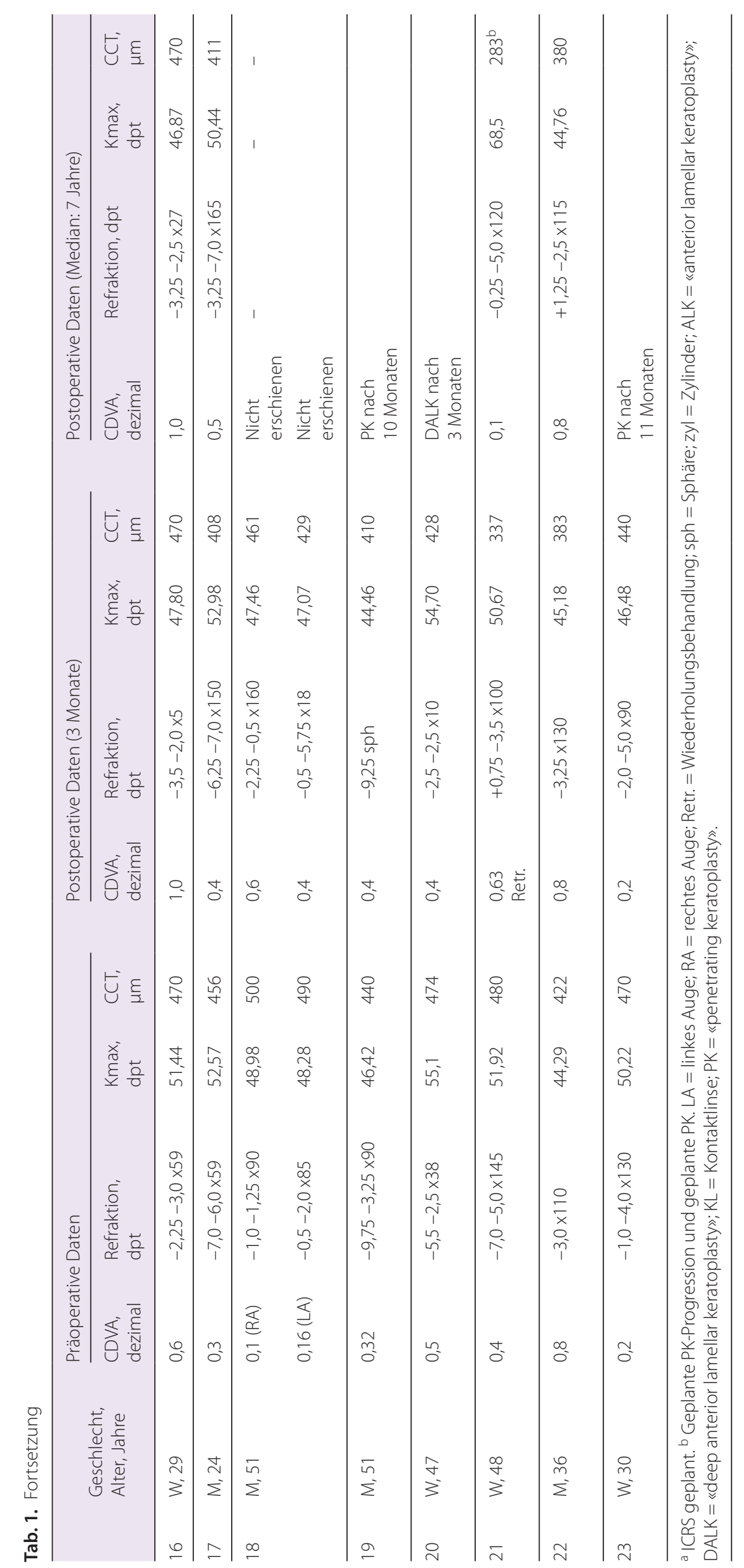


Tab. 2. Veränderungen der Sehschärfe und der Refraktion

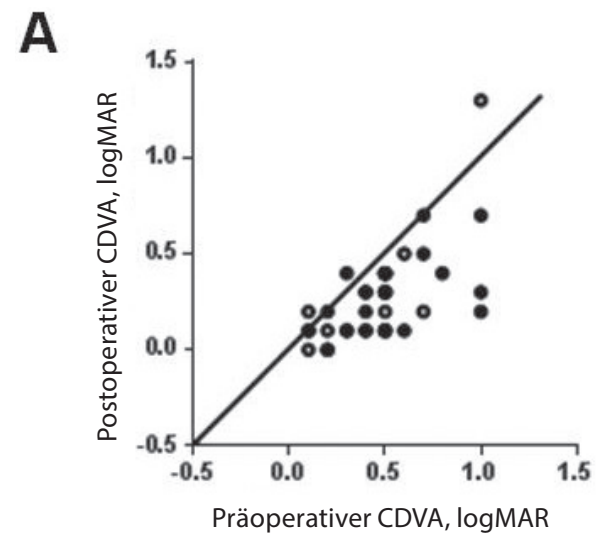

\begin{tabular}{llll}
\hline & Präoperativ & 3 Monate postoperativ & $\begin{array}{l}\text { Langzeit- } \\
\text { Nachuntersuchung }\end{array}$ \\
\hline CDVA, logMAR & $0,49 \pm 0,27$ & $0,27 \pm 0,18$ & $0,24 \pm 0,28$ \\
SÄ, dpt & $6,2 \pm 3,7$ & $3,7 \pm 3,1$ & $2,1 \pm 2,8$ \\
Zylinder, dpt & $-4,2 \pm 1,9$ & $-3,0 \pm 1,8$ & $-3,0 \pm 1,7$ \\
\hline
\end{tabular}

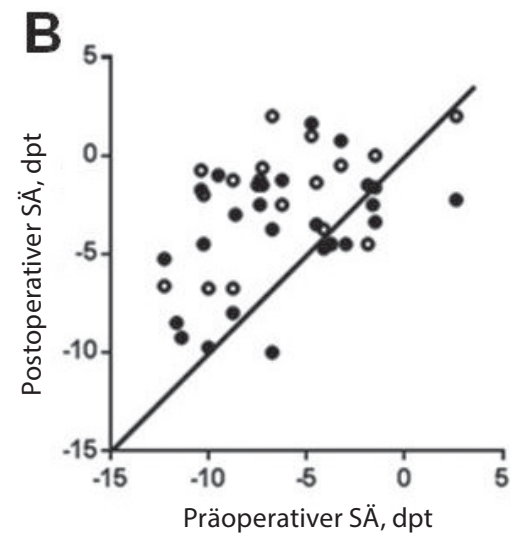

Abb. 2. a Der CDVA (logMAR) vor sowie 3 Monate und mehrere Jahre (langfristig) nach topografiegeführter PRK wegen Keratokonus. b Prä- und postoperative SÄ-Refraktion (dpt). c Prä- und postoperativer Zylinder (dpt). Ungefüllte Kreise = Nachuntersuchung nach 3 Monaten; gefüllte Kreise = Langzeit-Nachuntersuchung.

Abb. 3. a CCT $(\mu \mathrm{m})$ vor sowie 3 Monate und mehrere Jahre (langfristig) nach topografiegeführter PRK wegen Keratokonus. b Prä- und postoperative Kmax (dpt) im Verlauf des Nachbeobachtungszeitraums. Gefüllte Kreise $=$ Nachuntersuchung nach 3 Monaten; ungefüllte Kreise $=$ LangzeitNachuntersuchung.

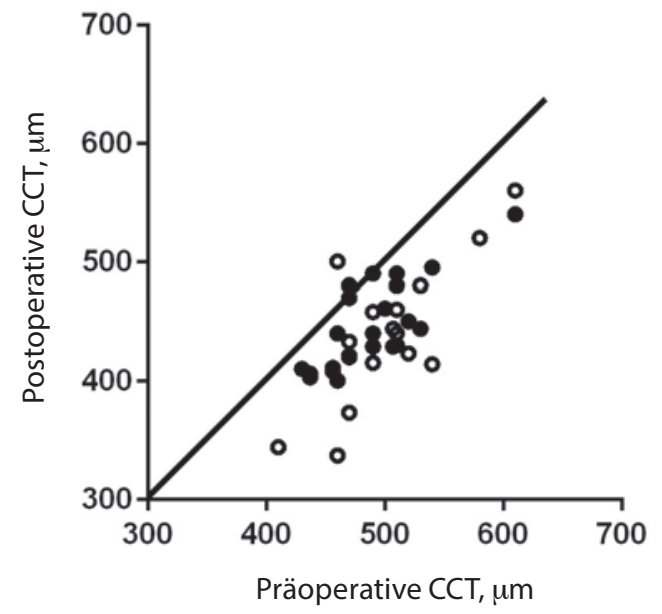

Vergleich hatten sich die SÄ-Werte von der Nachuntersuchung nach 3 Monaten bis zur Langzeit-Nachuntersuchung nicht signifikant verändert ( $p>0,05)$. Der mittlere refraktive Astigmatismus wurde signifikant reduziert: um 1,2 dpt sowohl nach 3 Monaten $(\mathrm{p}<0,05)$ als auch bei der Langzeit-Nachuntersuchung $(\mathrm{p}<0,05$; Tab. 2, Abb. 2c).

\section{Veränderung der Hornhaut-Dicke}

Die mittlere CCT betrug präoperativ $484 \mu \mathrm{m}$ (SA $45 \mu \mathrm{m}$; Bereich 410-610 $\mu \mathrm{m}$ ). Die CCT ging signifikant auf $440 \mu \mathrm{m}$ (SA $43 \mu \mathrm{m}$; Bereich 337-540 $\mu \mathrm{m}$ ) bei der Nachuntersuchung nach 3 Monaten und auf $434 \mu \mathrm{m}$ (SA $57 \mu \mathrm{m}$; Bereich 337-560 $\mu \mathrm{m}$ ) bei der LangzeitNachuntersuchung zurück ( $\mathrm{p}<0,05$; Abb. 3a).
Veränderungen in der Keratometrie

Kmax wurden anhand der steilsten Keratometermessung beurteilt. Der mittlere Kmax-Wert betrug präoperativ 50,6 dpt (SA 3,5 dpt; Bereich 44,29-57,89 dpt) und postoperativ 48,92 dpt (SA 3,0 dpt; Bereich 43,4-53,8 dpt) bei der Nachuntersuchung nach 3 Monaten und 49,3 dpt (SA 3,3 dpt; Bereich 44,8-56,6 dpt) bei der Langzeit-Nachuntersuchung. Somit waren die KmaxWerte nach 3 Monaten um 1,7 dpt gesunken $(\mathrm{p}<0,05)$ und nach im Median 7 Jahren um 1,3 dpt ( $p>0,05$; Abb. 3b).

\section{Hornhaut-Trübung}

Nach 3 Monaten lag in 20 Augen eine Trübung vom Schweregrad 1-2 und in 6 Augen vom Grad 3-4 vor. Bei der Langzeit-Nachuntersuchung zeigten 3 Augen keine Trübung, 15 Augen eine Trübung vom Grad 1 und 1 Auge eine Trübung vom Grad 2. 


\section{Diskussion}

Die Pathogenese des Keratokonus ist nicht im Detail bekannt, aber Nielsen et al. [8] haben festgestellt, dass im Hornhaut-Epithel eine veränderte Proteinexpression vorliegt, die mit Veränderungen des Zytoskeletts, herabgesetztem Remodeling der extrazellulären Matrix, veränderter Transmembran-Signalübermittlung und veränderter Zell-Zell- und Zell-Matrix-Interaktion einhergeht. Meek et al. [9] berichteten, dass die interne Kollagenstruktur der Hornhaut sich nach einem PRK-Eingriff langfristig verändert, was zu einer Hornhaut-Verdünnung und -Ektasie führt. Cennamo et al. [6] verzeichneten bei 25 behandelten Augen über einen Nachbeobachtungszeitraum von 24 Monaten einen signifikanten Rückgang bei allen Keratokonusindizes, während bei den unbehandelten Kontrollaugen die Indizes stiegen, was auf eine potenzielle therapeutische Wirkung der ExcimerLaser-Behandlung bei Keratokonus hindeutet. Guedj et al. [7] führten bei 62 Augen von 42 Patienten mit Verdacht auf Keratokonus eine PRK durch und beobachteten eine signifikante Verbesserung der Sehschärfe, die über einen Beobachtungszeitraum von bis zu 5 Jahren Bestand hatte. Chelala et al. [10] berichteten ebenfalls, dass die PRK bei gering- bis mittelgradigem Keratokonus ein sicheres und wirksames Verfahren zur Verbesserung des unkorrigierten Visus bei Patienten mit leichtem Refraktionsfehler darstellt. Kasparova und Kasparov [11] hingegen beobachteten eine Progression des Keratokonus in 8,6\% der Augen in den ersten 6 Monaten nach dem Eingriff. Koller et al. [12] plädierten für ein Monitoring mittels Hornhaut-Topografie von bis zu 5 Jahren, um sicherzugehen, dass ein PRK-Eingriff nicht zur Keratokonusprogression führt, nachdem die betreffende Hornhaut laut Dokumentation präoperativ seit Jahren stabil war. Tatsächlich kann eine Hornhaut-Ektasie sogar noch 10 Jahre nach einer PRK auftreten [13]. Darüber hinaus haben ICRS gegenüber der PRK den Vorteil, dass sie ein reversibles Verfahren zur Behandlung des gering- bis mittelgradigen Keratokonus darstellen. Allerdings ist die Stabilität des Verfahrens auf sehr lange Sicht weiterhin nicht geklärt [14].

In der hier beschriebenen Fallserie lag in allen Augen ein manifester Keratokonus vor, und die Behandlung erfolgte ohne Zusatztherapien wie die Kollagenvernetzung, die zur Zeit der Behandlung der meisten dieser Augen noch nicht Bestandteil der klinischen Praxis war. Gegenstand unserer Untersuchung waren Fälle, in denen die topografiegeführte PRK mit dem Ziel durchgeführt worden war, Myopie und Astigmatismus zu reduzieren und den unkorrigierten und bestkorrigierten Visus zu verbessern.
In dieser Untersuchung wurde die Keratokonusprogression im Median 7 Jahre nach einer PRK (Bereich 3-10 Jahre) betrachtet. Signifikante Verbesserungen des Sehvermögens lagen bei $84 \%$ der Patienten vor, die an der Langzeit-Nachuntersuchung teilnahmen, und bei 79\% dieser Patienten war keine Progression festzustellen. Das allgemeine Progressionsrisiko in unserer Kohorte betrug 25\% (6 von 24 Augen). Wir stellten außerdem fest, dass das mediane Alter zum Zeitpunkt der PRK bei 3 Patienten mit Keratokonusprogression rund 47 Jahre betrug. Eine Progression in so hohem Alter ist sehr selten, insofern könnte der PRK-Eingriff, der mit einer zusätzlichen Ausdünnung der Hornhaut einhergeht, ein initiierender Faktor für das Fortschreiten der Krankheit bei Patienten höheren Alters sein.

Da alle 23 Patienten ursprünglich für eine Hornhaut-Transplantation überwiesen worden waren, könnte die Studie auch darauf hindeuten, dass 4 von 5 Patienten mit Kontaktlinsen-Unverträglichkeit und gering- bis mittelgradigem Keratokonus eine Transplantation vermeiden können, indem sie sich einer PRK unterziehen. Darüber hinaus gaben mindestens 3 von 5 Patienten bei der Langzeit-Nachuntersuchung an, mit der Behandlung zufrieden zu sein. Abschließend ist zu sagen, obwohl ein Keratokonus allgemein als absolute Kontraindikation für eine PRK gilt, deuten unsere Ergebnisse darauf hin, dass die topografiegeführte PRK eine sinnvolle Option zur Reduktion von Myopie oder Astigmatismus und zur Verbesserung der Sehschärfe in ausgewählten Augen mit Keratokonus darstellen könnte. In der hier vorgestellten Studie wurde ein begrenztes Maß an Progression des Keratokonus nach einer PRK festgestellt.

\section{Danksagung}

Die Autoren danken Nicolaj Aagaard, Jens Christian Hedegaard, Henrik Sejersen und Christina Møller für ihre wertvolle Mitarbeit bei den klinischen Langzeit-Kontrolluntersuchungen.

\section{Ethikerklärung}

Die Studie wurde gemäß den Grundsätzen der Deklaration von Helsinki durchgeführt, und alle Teilnehmer erteilten vor der Teilnahme schriftlich ihr Einverständnis nach Aufklärung. Der Ausschuss für Ethik in der medizinischen Forschung der Region Mitteljütland (Videnskabsetiske Komitéer for Region Midtjylland) hat die Studie als Qualitätskontrollstudie eingestuft, für die nach dänischem Recht keine formelle Genehmigung durch eine Ethikkommission erforderlich ist.

\section{Disclosure Statement}

Die Autoren haben keine finanziellen Verbindungen offenzulegen.

\section{Referenzen}

1 Vazirani J, Basu S: Keratoconus: current perspectives. Clin Ophthalmol 2013;7:2019-2030.

$\checkmark 2$ Fadlallah A, Dirani A, EI Rami H, Cherfane G, Jarade E: Safety and visual outcome of Visian toric ICL implantation after corneal collagen cross-linking in keratoconus. J Refract Surg 2013;29:84-89.
3 Schmitt-Bernard CF, Lesage C, Arnaud B: Keratectasia induced by laser in situ keratomileusis in keratoconus. J Refract Surg 2000;16: 368-370.

4 Dawson DG, Randleman JB, Grossniklaus HE, et al: Corneal ectasia after excimer laser keratorefractive surgery: histopathology, ultra- structure, and pathophysiology. Ophthalmology 2008;115:2181-2191.

5 Bilgihan K, Ozdek SC, Konuk O, Akata F Hasanreisoglu B: Results of photorefractive keratectomy in keratoconus suspects at 4 years. J Refract Surg 2000;16:438-443. 
-6 Cennamo G, Intravaja A, Boccuzzi D, Marotta G, Cennamo G: Treatment of keratoconus by topography-guided customized photorefractive keratectomy: two-year follow-up study. J Refract Surg 2008;24:145-149.

7 Guedj M, Saad A, Audureau E, Gatinel D: Photorefractive keratectomy in patients with suspected keratoconus: five-year follow-up. J Cataract Refract Surg 2013;39:66-73.

8 Nielsen K, Birkenkamp-Demtröder K, Ehlers $\mathrm{N}$, Orntoft TF: Identification of differentially expressed genes in keratoconus epithelium analyzed on microarrays. Invest Ophthalmol Vis Sci 2003;44:2466-2476.
-9 Meek KM, Tuft SJ, Huang Y, et al: Changes in collagen orientation and distribution in keratoconus corneas. Invest Ophthalmol Vis Sci 2005;46:1948-1956.

$\checkmark 10$ Chelala E, Rami HE, Dirani A, Fadlallah A, Fakhoury O, Warrak E: Photorefractive keratectomy in patients with mild to moderate stable keratoconus: a five-year prospective followup study. Clin Ophthalmol 2013;7:1923-1928. 11 Kasparova EA, Kasparov AA: Six-year experience with excimer laser surgery for primary keratoconus in Russia. J Refract Surg 2003; 19(2 suppl):S250-S254.
12 Koller T, Iseli HP, Donitzky C, Ing D, Papadopoulos N, Seiler T: Topography-guided surface ablation for forme fruste keratoconus. Ophthalmology 2006;113:2198-2202.

13 Kim H, Choi JS, Joo CK: Corneal ectasia after PRK: clinicopathologic case report. Cornea 2006;25:845-848.

14 Piñero DP, Alio JL: Intracorneal ring segments in ectatic corneal disease - a review. Clin Experiment Ophthalmol 2010;38:154-167. 